logical point of view. The book is invaluable to all interested in the natural history of Africa, and is especially important as indicating the number of game animals to be met with in British territories and dependencies.

R. L.

An Introduction to the Carbon Compounds. By R. H. Adie, M.A., B.Sc. Pp. viii + 90. (London: W. B. Clive.)

WiTHIN the brief compass of this work the author aims at introducing the student to some of the chief groups of the carbon compounds, as represented by familiar substances, and at the same time at providing a series of experiments to illustrate the properties and reactions of these compounds. Thus the subject of the hydrocarbons is developed from an examination of the properties of coal gas, which leads to the study of marsh gas, ethane, olefine, acetylene and benzene. A feature of the book is that aromatic compounds are described along with fatty derivatives belonging to the same group, phenol along with alcohol, benzoic and salicylic acids along with acetic acid, aniline along with ethylamine, \&c. This arrangement of the matter produces, no doubt owing to the severe compression, a somewhat disconnected effect, as it in many cases prevents a complete and logical discussion of the constitution of the compounds which are mentioned. This renders the book less suitable for absolute beginners than for students who have already a slight elementary acquaintance with the subject, and to these it cannot fail to afford valuable assistance. The experiments are on the whole well selected, but they are conducted on purely qualitative lines, no attention being paid to that important factor - the yield.

A. $\mathrm{H}$.

\section{LETTER TO THE EDITOK.}

[The Editor does not hold himself responsible for opinions ex. pressed by his correspondents. Neither can he undertake to return, or to correspond with the woriters of, rejected manuscripts intended for this or any other part of NATURE. No notice is taken of anonymous communications.]

On the Deduction of Increase-Rates from Physical and other Tables.

Prof. Perry has called my attention to a want which sometimes arises in making practical deductions from tables. Take the following example.

\begin{tabular}{c|c|c|c|c}
\hline$\theta^{\circ} \mathrm{C}$. & $p$ & $\Delta p$ & $\Delta^{2} p$ & $\Delta^{3} p$ \\
\hline 90 & 1463 & & & \\
95 & 1765 & 302 & & \\
100 & 2116 & 351 & 49 & $\mathbf{5}$ \\
105 & 2524 & 408 & 62 & 5 \\
I10 & 2994 & 470 & 70 & 8 \\
I15 & 3534 & 540 & 78 & 8 \\
120 & 4152 & 618 & 78 & \\
\hline
\end{tabular}

The table gives in the second column the pressure of steam for the temperatures stated in the first column, which proceed by equal steps of $5^{\circ}$. The question is, how best to derive from these data the value of $\frac{d p}{d \theta}$ at one of the stated temperatures, say $105^{\circ}$.

The column $\Delta p$ gives the differences between consecutive values of $p$. The column $\Delta^{2} p$ gives the differences between consecutive values of $\Delta p$, and so on. The third differences $\Delta^{3} p$ exhibit so much irregularity that it is not worth while to proceed to fourth differences.

It is obvious that the required result is greater than $\frac{1}{5}$ of 408 , and less than $\frac{1}{5}$ of 470 . Half the sum of these two is a fair first approximation. Closer approximations can be obtained by means of the numbers printed in large type. Let the downward sloping series $470,70,8$ be called $d_{1} d_{2} d_{3}$, and the upward sloping series $408,57,8$ be called $u_{1} u_{2} u_{3}$. Also let the common difference $5^{\circ}$ be denoted by $h$.

NO. I $55 \mathrm{I}$, vOL. 60 ]
It is known to mathematicians that $h \frac{d p}{d \theta}$ is theoretically equal to $d_{1}-\frac{1}{2} d_{2}+\frac{1}{3} d_{3}-\& \mathrm{c}$., and also to $u_{1}+\frac{1}{2} u_{2}+\frac{1}{3} u_{3}+\& \mathrm{c}$., both series being supposed to be continued till we reach an order of differences that vanishes.

In physical tables, usually no column of differences vanishes exactly, and the two series will not exactly agree. The question is, how to get the best practical approximation out of them. The most obvious plan is to add them, and write

$$
2 h \frac{d p}{d \theta}=\left(d_{1}+u_{1}\right)-\frac{1}{2}\left(d_{2}-u u_{2}\right)+\frac{1}{3}\left(d_{3}+u_{3}\right)-\& \mathrm{c} \text {, , }
$$

then to take the first bracketed expression, the first two, the first three, \&c., as first, second, third, \&c., approximations. But it will be found on trial, in the present instance and in most instances, that the second approximation so obtained is less exact than the first.

I find, on looking into the matter strictly, that the prope second approximation is

$$
2 h \frac{d p}{d \theta}=\left(d_{1}+u_{1}\right)-\frac{1}{6}\left(d_{2}-u_{2}\right) \text {. }
$$

This equation would be exact if $p$ were capable of being expressed in the form

$$
p=\mathrm{A} \theta+\mathrm{B} \theta^{2}+\mathrm{C} \theta^{3}+\mathrm{D} \theta^{4}
$$

As applied to the example before us, it gives 87.7 as the value of $2 h \frac{d p}{d \theta}$, and 8.77 as the value of $\frac{d p}{d \theta}$. This is as close an approximation as is warranted by the data. The first approximation $\left(d_{1}+u_{1}\right)$ is $87 \cdot 6$.

The two series $d_{1}-\frac{1}{2} d_{2}+\& c$. , and $u_{1}+\frac{1}{2} u_{2}+\& c$., carried each to three terms, give respectively 87.53 and $87 \cdot 83$.

The proper third approximation, which would be exact for is

$$
p=\mathrm{A} \theta+\mathrm{B} \theta^{2}+\mathrm{C} \theta^{3}+\mathrm{D} \theta^{4}+\mathrm{E} \theta^{5}+\mathrm{F} \theta^{6},
$$

$$
2 h \frac{d p}{d \theta}=\left(d_{1}+u_{1}\right)-\frac{1}{8}\left(d_{2}-u_{2}\right)+\frac{1}{3 \sigma}\left(d_{3}+\varepsilon_{3}\right) .
$$

Another requisite is to determine $\frac{d^{2} p}{d \theta^{2}}$. When the fourth ordey of differences vanishes, I find that $d_{1}-u_{1}$ is the accurate value of $h^{2} \frac{d^{2} p}{d \theta^{2}}$. In the present instance this gives

$$
\frac{d^{2} p}{d \theta^{2}}=\frac{62}{25}=2 \cdot 48
$$

The formulæ most employed hitherto for this purpose are

$$
\begin{aligned}
h^{2} \frac{d^{2} p}{d \theta^{3}} & =d_{2}-d_{3}+\frac{\mathrm{II}}{\mathrm{I} 2} d_{4}-\& \mathrm{c} . \\
& =u_{2}+u_{3}+\frac{\mathrm{II}}{\mathrm{I} 2} u_{4}+\& \mathrm{c} .
\end{aligned}
$$

which, if we include two terms of each, give respectively $\frac{62}{25}$ and $\frac{65}{25}$.

When fourth and fifth differences are worthy of attention, the: correction to be made for them consists in adding

$$
\frac{1}{5}\left(d_{1}-u_{1}\right)-\frac{1}{12}\left(d_{2}+u_{2}\right)
$$

to the first approximation $d_{1}-\imath \iota_{1}$.

To take account of fifth and sixth differences, this correction. must be supplemented by a further addition of

$$
\frac{1}{15}\left(d_{1}-u_{1}\right)-\frac{1}{3} \delta\left(d_{2}+u_{2}\right)+\frac{1}{9} \sigma\left(d_{3}-2 \sigma_{3}\right) \text {. }
$$

Without occupying space by a detailed investigation, I may say that my plan of procedure is first to write down (by Taylor's. theorem) the expansions for the first differences in ascending powers of $h$; then so to combine them in pairs by subtraction as to eliminate all even powers; then to eliminate $h^{3}$ from two. of the resulting equations. This gives.

when $h^{5}$ is neglected.

$$
2 h \frac{d p}{d \theta}=\left(d_{1}+u_{1}\right)-\frac{1}{6}\left(d_{2}-u u_{2}\right)
$$

The next approximation is obtained by eliminating both $h^{3}$ and $h^{5}$ from three of the equations.

In the first operation for deducing $\frac{d^{2} p}{d \theta^{2}}$ the pairs are combined by addition instead of subtraction, thus eliminating all odd powers of $h$.

This gives $d_{1}-u_{1}=h^{2} \frac{d^{2} p}{d \theta^{2}}$ when $l^{4}$ is negligible. The succeeding approximations are obtained by eliminating first $h^{+}$and then both $h^{4}$ and $h^{6}$.
J. D. EVERETT. 